\title{
El Entramado Temático de la Comunicación Organizacional: nitidez, cohesión y posicionamiento en la red conceptual de las publicaciones sobre comunicación en el SciELO Citation Index
}

\author{
Alejandro García-Macías ${ }^{1 *}$, Sarahí Orduño Mendoza ${ }^{2}$, Betsabee Fortanell Trejo ${ }^{3}$
}

\begin{abstract}
Resumen
La comunicación organizacional está posicionada como un subcampo bien delimitado en la estructura temática del campo de la comunicación en América Latina, al desarrollar temas centrales bien definidos y cercanamente vinculados con otros tópicos clásicos y emergentes transversales en la disciplina. No obstante, la producción científica del subcampo evidencia elementos de centralización editoriales, nacionales e institucionales. Así se desprende de la identificación y análisis del Entramado Temático de la Comunicación Organizacional, a partir de la red de co-ocurrencia de palabras clave en los artículos de investigación sobre comunicación publicados en SciELO Citation Index entre 1997 y 2017.

Abstract

Organizational communication is currently positioned as a well delimited subfield within the thematic structure of the Communication field in Latin America, since it develops well defined core issues that are closely tied to other classic and emergent crosscutting topics in the discipline. Nevertheless, the subfield scientific production shows centralization characteristics according to editorial, national and institutional criteria. These conclusions emerge from the identification and further analysis of the Thematic Interweaving of Organizational Communication, in turn derived from a co-ocurrence network of the keywords in the research articles on Communication published in SciELO Citation Index between 1997-2017.
\end{abstract}

Palabras Clave

Comunicación Organizacional, Entramado temático, Campo académico de la comunicación, Análisis de redes sociales, Bibliometría

Keywords

Organizational Communication, Thematic Interweaving, Academic Field of Communication, Social Network Analysis, Bibliometrics

${ }^{1}$ Doctor en Sociología. Departamento de Comunicación, Universidad Autónoma de Aguascalientes (UAA), México

${ }^{2}$ Licenciada en Comunicación Organizacional. Maestría en Investigaciones Sociales y Humanísticas, UAA

${ }^{3}$ Maestra en Comunicación. Doctorado en Estudios Socioculturales, UAA

*Autor para correspondencia: alejandro.garciam@edu.uaa.mx

\section{Una mirada reticular al campo de la comunicación en América Latina}

El objetivo del estudio que se reporta consistió en identificar y analizar las temáticas predominantes en la investigación en comunicación organizacional durante las últimas décadas en América Latina, así como en determinar las formas en que estos temas se vinculan y articulan entre ellos y con otros múltiples tópicos abordados en el campo de la comunicación.

Un ejercicio de esta naturaleza es pertinente cuando se intenta abonar a la reflexión en torno a las particularidades 
del campo, que como ha sido bien documentado, en América Latina se caracteriza por una amplia "dispersión temática y teórico-metodológica”, la que además parece prevalecer como tendencia en todo el mundo y que "coexiste con una creciente concentración de la producción y la distribución de contribuciones científicas, sin que esto signifique una paradoja o una contradicción" (Fuentes-Navarro, 2016, pp. 112-113).

El análisis del Entramado Temático de la Comunicación Organizacional que se describe se enmarca en un proyecto más amplio, de tipo bibliométrico, que se propuso observar y describir las estructuras relacionales del campo a partir de la investigación que en él se genera, fundamentalmente (1) las de colaboración directa entre investigadores, instituciones o países, a través de las co-autorías; (2) las de citas académicas, que evidencian procesos de diálogo, influencia, etc., entre autores o grupos a través de sus textos, mediante un análisis de citas directas, co-citaciones y acoplamiento bibliográfico y; (3) las temáticas o conceptuales, entendidas como redes semánticas que representan relaciones entre palabras o conceptos en un conjunto de publicaciones, por medio de un estudio de co-ocurrencia de sus palabras clave (García-Macías \& Fortanell, 2018).

Los datos consistieron en un conjunto de registros bibliográficos provenientes del SciELO Citation Index de la Web of Science. En buena medida, esta base de datos proporciona un panorama razonable de la productividad científica latinoamericana, dado que contiene revistas de al menos 13 países de la región (además de España, Portugal y Sudáfrica) y que su modelo fue "especialmente desarrollado para responder a las necesidades de la comunicación científica en los países en desarrollo y particularmente de América Latina y el Caribe" (SciELO, n.d.).

La base de registros se limitó a los textos catalogados como artículos de investigación publicados entre 1997 y 2017, que incluyeran el término Comunicación en el tema y estuviesen indexados bajo la categoría Communication. Con ello se identificaron 936 artículos, publicados por 16 revistas latinoamericanas distintas, de la autoría de 1,175 autores adscritos a 404 instituciones en 31 países. La base de datos consta también de 20,573 referencias bibliográficas, 15,199 fuentes y 13,376 primeros autores citados, además de 2,610 palabras clave establecidas por los propios autores (García-Macías \& Fortanell, 2018).
Una parte de dicho proyecto, más amplio, se concentró en reflejar la estructura intelectual del campo y delinear un mapa de sus principales temas de interés (Khasseh, Soheili, Moghaddam, \& Chelak, 2017), haciendo uso del Análisis de Redes Sociales (ARS). El ARS se define como un conjunto de métodos para el análisis de estructuras sociales (Degene \& Forsé, 1999; Scott, 2000), en el que los vínculos o relaciones entre entidades son las unidades básicas de recogida de información y de análisis, en lugar de los datos atributivos clásicos de los individuos (Lozares, 1996); esto es, que los actores y sus relaciones se entienden siempre como interdependientes, en lugar de como unidades independientes y autónomas (Wasserman \& Faust, 1994).

Una red social es una estructura compuesta por un conjunto de actores, algunos de los cuales están conectados a través de una o más relaciones (Knoke \& Yang, 2008; Lozares, 1996). Conforme a la teoría de grafos, los actores se denominan y representan como nodos y los vínculos entre ellos como vértices. Gráficamente, nodos y vértices pueden asumir propiedades visuales (e.g., forma, tamaño, color, grosor de las líneas) que representen sus atributos categóricos (e.g., sexo, importancia, intensidad, tipo o dirección de la relación).

Adoptar este enfoque permitió superar las aproximaciones más tradicionales en bibliometría, que regularmente se basan en conteos simples de frecuencias de términos o en otras métricas de evaluación de la producción científica, mientras que ignoran otros aspectos relacionales clave, que pueden ser mejor analizados desde una lógica de redes (Montoya, Alcayde, Baños, \& Manzano-Agugliaro, 2018; Zhao, Mao, \& Lu, 2018). En el caso descrito en este artículo, además de conocer cuáles temas, cuántas veces y bajo qué enfoques han sido trabajados, fue posible identificar vínculos, afinidades, núcleos, exclusiones y otros patrones estructurales generales, subyacentes y latentes que muestran con mayor exactitud la configuración y características distintivas de un campo.

Algunos autores han denominado a esta técnica como "mapeo bibliométrico" por basarse en representaciones espaciales de cómo las disciplinas, campos, especialidades, y documentos o autores, están relacionados unos con otros (Gutiérrez-Salcedo, Martínez, Moral-Munoz, Herrera-Viedma, \& Cobo, 2017) aunque, desde la óptica de los autores de este artículo, tal denominación es al 


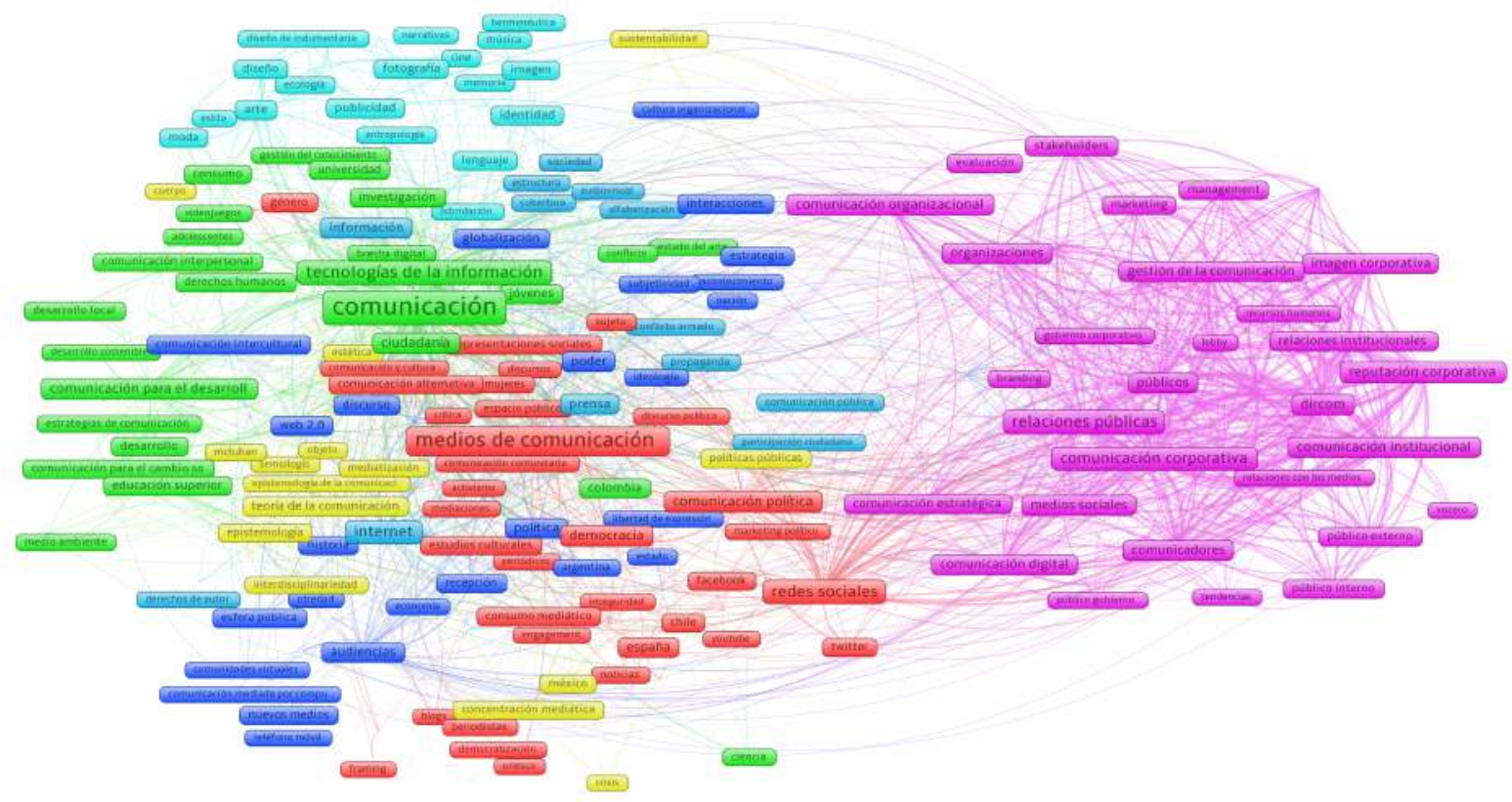

A voswiewer

Figura 1. Red completa de palabras clave sobre el tema Comunicación, con un mínimo de tres ocurrencias en los 936 artículos de la base de datos $(\mathrm{n}=286)$. Los clústeres se identifican por color y el tamaño de la etiqueta de los nodos refleja su frecuencia de ocurrencia.

menos parcial e incompleta, puesto que el ARS no se agota en el simple proceso de mapeo o visualización de gráficos, sino que ofrece múltiples métricas relacionales complejas, desarrolladas tanto desde la propia teoría de grafos como desde el álgebra matricial ${ }^{1}$. En este texto se utilizan únicamente tres de las métricas más intuitivas: las centralidades de grado, cercanía e intermediación (Freeman, 1979).

Porque, si bien "la visualización es una importante herramienta para la exploración de los datos, ayudando a los investigadores a tamizar los datos, detectar patrones interesantes y darle sentido a las observaciones" (Brandes, Kenis, \& Raab, 2005, p. 16), en ocasiones el ARS se presenta bajo la reducción a una "caja de herramientas de naturaleza técnico-formal y/o gráfica en la búsqueda pragmatista y eficaz de resultados, sin que se dé una reflexión suficiente sobre las condiciones de recogida de información o sobre la naturaleza de los datos y su contextualización o sobre las dimensiones

\footnotetext{
${ }^{1}$ Para un compendio de estas medidas ver, por ejemplo, Hanneman \& Riddle, 2005.
}

cognitivas, fácticas, dinámicas, simbólicas, etc., que supone la relación social o sobre los campos en los que se insertan dichas relaciones" (Lozares, 2005, p. 9).

Lo que aquí se denomina como el Entramado Temático de la Comunicación Organizacional es una red conceptual o de co-palabras. Estas son redes dadas por relaciones de co-ocurrencia, es decir, por la aparición conjunta de dos o más palabras en el título, resumen o listado de palabras clave de los documentos en un conjunto de publicaciones (van Eck \& Waltman, 2014). Dichas relaciones (o correlaciones) pueden ser más o menos fuertes o intensas, dependiendo de la frecuencia de tales apariciones conjuntas (Liu, Hu \& Wang 2012, citados en Khasseh et al., 2017).

Las visualizaciones se produjeron con el software VOSviewer $^{2}$, que se basa en un algoritmo de mapeo orientado a la visualización de similitudes, en el que cada nodo es agrupado dentro de un clúster de otros nodos cercanamente relacionados, generando grafos tanto tradicionales como de

${ }^{2}$ Desarrollado en la Universidad de Leiden, Holanda. www.vosviewer.com 
Tabla 1. Indicadores bibliométricos primarios de las palabras clave del Clúster Conceptual de Comunicación Organizacional

\begin{tabular}{lrrrlrrr}
\hline \multicolumn{1}{c}{ Palabra Clave } & Ocr. ${ }^{1}$ & Vnc. $^{2}$ & Frt. $^{3}$ & \multicolumn{1}{c}{ Palabra Clave } & Ocr. & Vnc. & Frt. \\
\hline Relaciones públicas & 41 & 69 & 363 & Resp. social empresarial & 10 & 45 & 115 \\
Comunicación corporativa & 36 & 60 & 345 & Marketing & 9 & 41 & 89 \\
Dircom & 25 & 51 & 326 & Management & 7 & 37 & 105 \\
Comunicación empresarial & 24 & 52 & 301 & Público externo & 7 & 35 & 102 \\
Comunicación organizacional & 21 & 55 & 125 & Público interno & 7 & 35 & 102 \\
Reputación corporativa & 21 & 49 & 271 & Evaluación & 7 & 8 & 8 \\
Comunicación institucional & 21 & 47 & 285 & Relaciones con la comunidad & 5 & 31 & 87 \\
Organizaciones & 18 & 55 & 146 & Branding & 5 & 25 & 36 \\
Públicos & 18 & 52 & 245 & Gobierno corporativo & 4 & 34 & 47 \\
Comunicadores & 17 & 45 & 195 & Lobby & 3 & 33 & 44 \\
Medios sociales & 17 & 53 & 191 & Liderazgo & 3 & 30 & 42 \\
Gestión de la comunicación & 16 & 48 & 197 & Relaciones con la prensa & 3 & 35 & 60 \\
Imagen corporativa & 15 & 46 & 201 & Relaciones con los medios & 3 & 35 & 60 \\
Comunicación digital & 15 & 47 & 159 & Recursos humanos & 3 & 31 & 54 \\
Comunicación estratégica & 13 & 47 & 93 & Tendencias & 3 & 26 & 39 \\
Marcas & 13 & 45 & 158 & Público gobierno & 3 & 21 & 34 \\
Comunicación interna & 11 & 45 & 68 & Vocero & 3 & 13 & 26 \\
Stakeholders & 11 & 42 & 114 & Identidad corporativa & 3 & 6 \\
Relaciones institucionales & 11 & 38 & 176 & & - & - \\
\hline
\end{tabular}

Fuente: Elaboración propia. Indicadores con base en la red total de palabras clave con un mínimo de tres ocurrencias ( $\mathrm{n}=286$ ) en los 936 artículos de la base de datos.

1 Número de ocurrencias

2 Número de vínculos con otras palabras clave en la red completa

3 Fortaleza de los vínculos

densidad y de superposición (van Eck \& Waltman, 2013, 2014; van Eck, Waltman, Dekker, \& van den Berg, 2010). Las métricas de red se calcularon con apoyo del software Visone ${ }^{3}$.

\section{Un clúster visualmente nítido, cohesivo y temáticamente coherente}

Para lograr una decantación de conceptos relevantes en la red conceptual del campo de la comunicación (2,610 palabras clave) se fijó un criterio de ocurrencia de palabras de nivel 3 , lo que significa que un término se incluye siempre y cuando aparezca como mínimo tres veces en el conjunto de artículos. Ello dio como resultado una red de 286 temas en la que se aprecian dos grandes conglomerados compuestos por siete clústeres de similitudes relacionales (Figura1). Como se percibe a la derecha del grafo, uno de estos dos conglomerados conforma por sí mismo un clúster claramente independiente.

Este clúster, compuesto por 37 conceptos, requiere mayor análisis en virtud de que las palabras que lo

\footnotetext{
${ }^{3}$ Generado por la Universidad de Konstanz, Alemania. www.visone.info
}

conforman se asocian notablemente con las áreas de interés de la comunicación organizacional, las asignaturas propias del subcampo y las denominaciones que recibe en diferentes contextos. En tal sentido, se procedió a aislar este conglomerado para identificar sus propiedades de composición y estructura; para efectos prácticos se le ha denominado el Clúster Conceptual de Comunicación Organizacional (Clúster CCO).

En la Tabla 1 se presentan los términos que lo conforman junto con tres indicadores primarios de su importancia al interior de la red completa: la frecuencia de ocurrencia (Ocr) o el número de veces que la palabra se incluye en los listados de palabras clave; el número de vínculos (Vnc) o la cantidad de otros términos con los cuales aparece en forma conjunta (solamente puede haber un vínculo entre cada par de términos), y; la fortaleza total de los lazos (Frt) o suma de las intensidades de todas las relaciones que mantiene con otros términos (e.g., si dos palabras aparecen simultáneamente una sola vez, la fortaleza será de uno; si lo hacen en tres ocasiones, será de tres). 


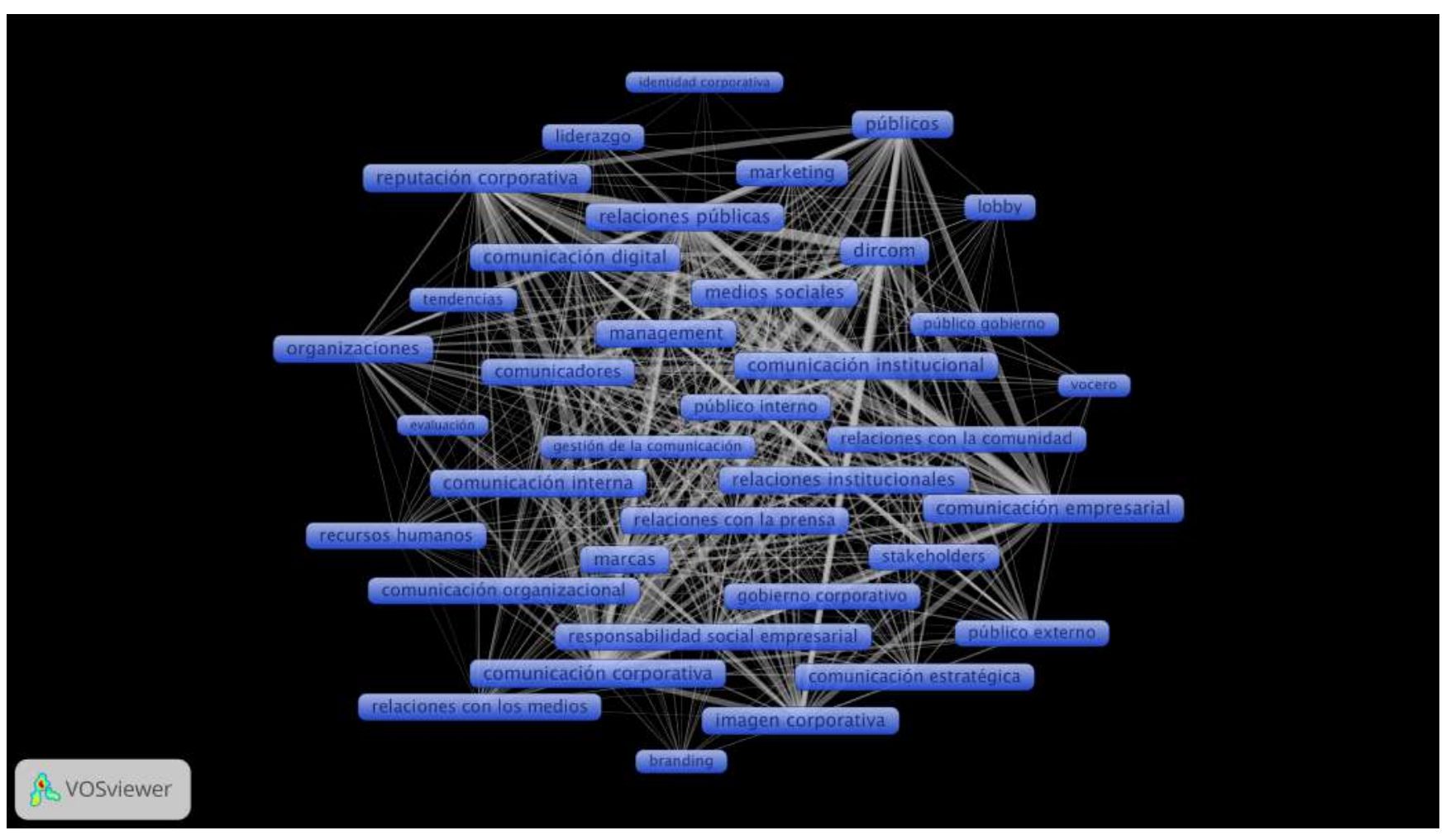

Figura 2. Sociograma del Clúster Conceptual de Comunicación Organizacional, aislado de la red completa.

Los dos últimos indicadores se ejemplifican mejor con el sociograma de la Figura 2, en donde los vértices que unen a las palabras del clúster se representan con un grosor diferente de acuerdo con la fortaleza de la co-ocurrencia. Las palabras clave Relaciones Públicas, Comunicación Corporativa, Dircom, Comunicación empresarial, Comunicación Organizacional, Reputación corporativa y Comunicación institucional concentran los mayores puntajes de Ocr, aunque a ellas se agregan otras con notable importancia conforme a su Vnc, tales como Organizaciones, Medios sociales y Públicos.

Es de destacarse que, aunque la palabra clave Comunicación organizacional aparece en 21 ocasiones (uno de los cinco valores más altos) como descriptora de los textos, al mismo tiempo tiene un puntaje relativamente muy bajo conforme a Frt (125, superada por quince conceptos). Ello significa que, pese a estar ligada a otras 55 palabras (el tercer valor más alto), el número de apariciones simultáneas con ellas es reducido y no es de alta recurrencia.

Las métricas de este grafo aislado de la red completa indican que se trata de una red conceptual muy cohesiva, con una densidad de .773 (donde 1 representaría una interconexión total entre los nodos) y un grado medio de 27.84 , lo que significa que, como media, cada concepto se relaciona directamente con cerca de 28 más, de un total de 36 vínculos posibles para cada nodo.

\section{La producción científica detrás del clúster: variedad y concentración}

Una segunda fase del estudio que aquí se describe, consistió en contextualizar los datos relacionales mediante el análisis de algunas características de la producción científica que les da origen. Para ello, la base de registros bibliográficos fue segmentada mediante la extracción de los 128 artículos que contienen las 37 palabras clave del Clúster CCO (ver Tabla 2).

Todos estos textos se publicaron entre 2005 y 2017, siendo 2011 y 2012 los años más prolíficos (26 y 21 artículos), mientras que los menos fueron 2005, 2008 (1) y 2006 (0). En los cinco años más recientes (2013-17) se publicaron en promedio 10 artículos por año. Los documentos corresponden 
Tabla 2. Artículos ( $\mathrm{n}=128)$ a partir de los que se conforma el Clúster Conceptual de Comunicación Organizacional en la red completa, según primer autor.

\begin{tabular}{|c|c|c|c|c|c|c|c|}
\hline \multicolumn{2}{|r|}{ Texto } & \multicolumn{2}{|r|}{ Texto } & \multicolumn{2}{|r|}{ Texto } & \multicolumn{2}{|r|}{ Texto } \\
\hline 1 & Anónimo (2010) & 33 & Garay Madariaga (2010) & 65 & Losada-Díaz (2015) & 97 & Pinzón-Rios (2015) \\
\hline 2 & Aguilera (2017) & 34 & García Montero (2017) & 66 & Mafra (2016) & 98 & Pol (2012) \\
\hline 3 & Álvarez-Nobell (2011) & 35 & García (2010) & 67 & Malizia (2012) & 99 & Prats (2013) \\
\hline 4 & Arango-Forero (2013a) & 36 & Gil Cubillos (2012) & 68 & Mancucci (2007) & 100 & Preciado-Hoyos (2015) \\
\hline 5 & Arango-Forero (2013b) & 37 & Gil Cubillos (2010) & 69 & Marca (2017) & 101 & Preciado-Hoyos (2014) \\
\hline 6 & Arango (2011) & 38 & Giraldo-Dávila (2011) & 70 & Martín Nieto (2007) & 102 & Preciado-Hoyos (2012) \\
\hline 7 & Arébalos (2010) & 39 & Giraldo-Dávila (2016) & 71 & Martín (2007) & 103 & Reyes (2012) \\
\hline 8 & Ávila (2016) & 40 & González López (2015) & 72 & Martínez Lahitou (2011) & 104 & Rodríguez Bravo (2011) \\
\hline 9 & Benavides (2017) & 41 & González-Luis (2014) & 73 & Mascio (2013) & 105 & Rodríguez-Polo (2013) \\
\hline 10 & Botero Escobar (2012) & 42 & González (2013) & 74 & Mazzola (2012) & 106 & Rüdiger (2014) \\
\hline 11 & Bouzon (2015) & 43 & Goyanes (2016) & 75 & Menanteau (2012) & 107 & Sabés Turno (2009) \\
\hline 12 & Briançon Messinger (2007) & 44 & Gutiérrez-García (2010) & 76 & Menéndez Echavarria (2013) & 108 & Salamanca Ávila (2005) \\
\hline 13 & Briançon Messinger (2009) & 45 & Gutiérrez (2011) & 77 & Monserrat-Gauchi (2014) & 109 & Salas Forero (2013) \\
\hline 14 & Brönstrup Silvestrin (2007) & 46 & Haase (2009) & 78 & Montaner Rodríguez (2011) & 110 & Salas Forero (2011) \\
\hline 15 & Cabra (2008) & 47 & Holzmann (2010) & 79 & Montoya (2009) & 111 & Salas González (2009) \\
\hline 16 & Carvalho (2016) & 48 & Hormazábal (2012) & 80 & Moreno (2014) & 112 & Salazar Gómez (2011) \\
\hline 17 & Cerezo (2013) & 49 & Iurcovich (2012) & 81 & Moya-Sánchez (2016) & 113 & Sanguine (2011) \\
\hline 18 & Cóppola (2012) & 50 & Jimenez (2011) & 82 & Muñoz Antonin (2010) & 114 & Scheinsohn (2010) \\
\hline 19 & Correa Ríos (2010) & 51 & Kröhling Kunsch (2007) & 83 & Muñoz-Navarro (2011) & 115 & Segura (2014) \\
\hline 20 & Costa-Sánchez (2014) & 52 & Kunsch (2012) & 84 & Navarro Ruiz (2012) & 116 & Soto-Vélez (2009) \\
\hline 21 & Cuervo (2011) & 53 & Labarca (2017) & 85 & Olabe Sánchez (2015) & 117 & Stortini (2011) \\
\hline 22 & de Souza Brandão (2015) & 54 & Lanza (2011) & 86 & Ontiveros (2013) & 118 & Suárez (2012) \\
\hline 23 & del Aguila Lima (2015) & 55 & Lasta (2013) & 87 & Orozco Toro (2015) & 119 & Tejeda García (2011) \\
\hline 24 & Di Pino (2011) & 56 & Lattuada (2011a) & 88 & Orozco Toro (2012) & 120 & Tuzzo (2011) \\
\hline 25 & Dueñas (2016) & 57 & Lattuada (2011b) & 89 & Orozco-Toro (2017) & 121 & Uribe (2017) \\
\hline 26 & Fernández Souto (2012) & 58 & Lattuada (2010a) & 90 & Ortiz Gonzales (2010) & 122 & Vahos Montoya (2009) \\
\hline 27 & Fernández Souto (2016) & 59 & Lattuada (2010b) & 91 & Otero (2012) & 123 & Varona (2007) \\
\hline 28 & Fernández-Cavia (2015) & 60 & Laura Guaita (2012) & 92 & Pagani (2012) & 124 & Veneziani (2015) \\
\hline 29 & Fernández (2014) & 61 & Lauro (2011) & 93 & Paladines galarza (2013) & 125 & Veneziani (2012) \\
\hline 30 & Ferrari (2012) & 62 & Lizarralde Gómez (2011) & 94 & Pedace (2011) & 126 & Venier (2016) \\
\hline 31 & Fofonca (2010) & 63 & Lojo (2011) & 95 & Perea Rodríguez (2013) & 127 & Yasky (2011) \\
\hline 32 & Fuentes Martínez (2007) & 64 & López (2016) & 96 & Pessoni (2011) & 128 & Zapata Cárdenas (2014) \\
\hline
\end{tabular}

Fuente: Elaboración propia. Extracción de los 128 artículos de la base de datos que contienen las 37 palabras clave del Clúster Conceptual de Comunicación Organizacional. El paquete informático utilizado (VosViewer) considera para fines analíticos solamente al primer autor de los documentos.

a 86 primeros autores distintos ${ }^{4}$, donde unos pocos publicaron más de un texto: Paola Lattuada (4), Jaime Alberto Orozco Toro y Ángela Preciado - Hoyos (3), Arango-Forero, Briançon Messinger, Fernández Souto, Gil Cubillos, Giraldo-Dávila, Salas Forero y Veneziani (2).

La Tabla 3 aporta datos sobre la institución de adscripción y los países de los autores, así como sobre las revistas que publicaron los artículos. Lo primero que destaca es un aspecto de orden institucional, ya que se detecta una correspondencia positiva entre la productividad de los autores según su adscripción y el número de publicaciones de las

\footnotetext{
${ }^{4} \mathrm{El}$ dato debe interpretarse con cautela en el sentido de posibles identificadores de autor múltiples, que son frecuentes en las bases de datos y en su análisis automatizado. No puede determinarse con certeza, a veces incluso ni mediante una revisión manual caso por caso, si dos nombres registrados pertenecen al mismo autor, por ejemplo, Arango (2011) y Arango-Forero (2013a).
}

revistas que son alojadas precisamente en esas instituciones. La concentración editorial e institucional es también evidente.

En lo alto de la tabla aparecen los autores de la Universidad de Palermo, en Argentina, con 13 documentos; a esta misma institución pertenece la revista que reúne el mayor número de artículos, Cuadernos del Centro de Estudios en Diseño y Comunicación (45, el 35\% del total). Es seguida por la Pontificia Universidad Javeriana, de Colombia, con 10 artículos, que aloja a Signo y pensamiento, la segunda revista con más documentos del clúster (22). El tercer sitio lo ocupa la Universidad de la Sabana, de Colombia (6 artículos, al igual que la UAB), que es la sede de la revista Palabra clave, también tercera en cuanto al número de artículos publicados.

Con relación a los países de los autores, nuevamente se advierte una fuerte centralización, ya que casi el $70 \%$ de la 
Tabla 3. Artículos $(n=128)$ a partir de los que se conforma el Clúster Conceptual de Comunicación Organizacional en la red completa, según institución de adscripción de los autores, país y revista de publicación.

\begin{tabular}{|c|c|c|c|c|c|}
\hline \multicolumn{2}{|l|}{ Documentos por Institución ${ }^{1}$} & \multicolumn{2}{|c|}{ Documentos por País } & \multicolumn{2}{|l|}{ Documentos por Revista } \\
\hline Universidad de Palermo & 13 & España & 29 & Cuadernos del Centro de Estudios en Diseño y Com. & 45 \\
\hline Pontificia Universidad Javeriana & 10 & Colombia & 25 & Signo y Pensamiento & 22 \\
\hline Universidad de la Sabana & 6 & Argentina & 20 & Palabra Clave & 18 \\
\hline Universitat Autònoma de Barcelona & 6 & Brasil & 15 & Intercom & 10 \\
\hline Pontificia Universidad Católica de Chile & 4 & Chile & 9 & Anagramas & 8 \\
\hline Universidad de Medellín & 4 & EE.UU & 3 & Cuadernos.info & 7 \\
\hline Universidad Pontificia Bolivariana & 4 & México & 3 & Punto cero & 6 \\
\hline Universidad de Buenos Aires & 3 & Bolivia & 2 & Observatorio (obs*) & 5 \\
\hline Universidad de Navarra & 3 & Ecuador & 2 & La trama de la comunicación & 3 \\
\hline Universidade de São Paulo & 3 & Italia & 2 & Comuni@cción & 1 \\
\hline Universidad Carlos III de Madrid & 2 & Alemania & 1 & Comunicación y Sociedad & 1 \\
\hline Universidad de Chile & 2 & Bélgica & 1 & Dixit & 1 \\
\hline Universidad de Ciencias Sociales y Empresariales & 2 & Cuba & 1 & Liberabit & 1 \\
\hline Universidad de Murcia & 2 & Francia & 1 & & \\
\hline Universidad de Vigo & 2 & Omán & 1 & & \\
\hline Universidad del Salvador & 2 & No Especif. & 23 & & \\
\hline Universidad Diego Portales & 2 & & & & \\
\hline Universidad Finis Terrae & 2 & & & & \\
\hline Universidad Rey Juan Carlos & 2 & & & & \\
\hline Universidade Federal Do Rio Grande Do Sul & 2 & & & & \\
\hline $\mathrm{UP}^{2}$ & 2 & & & & \\
\hline No Especificado & 20 & & & & \\
\hline Total $^{3}$ & 98 & Total $^{3}$ & 138 & Total & 128 \\
\hline
\end{tabular}

producción científica del clúster se concentra en cuatro países ${ }^{5}$ : España (29 artículos), Colombia (25), Argentina (20) y Brasil (15). El papel de los autores españoles se explica tanto por su participación directa en las publicaciones, como por las relaciones colaborativas institucionales evidenciadas por la presencia de universidades como la Autónoma de Barcelona, de Navarra, Carlos III de Madrid y de Murcia, entre otras.

\section{Las relaciones entre clústeres temáticos: detectar un entramado}

Como ya se ha indicado, los 37 términos que conforman el Clúster CCO se asignaron en forma automática a dicho componente con base en el algoritmo de visualización de similitudes. Posteriormente, este clúster se analizó en forma aislada, con lo que deliberadamente se excluyó el resto de los conceptos pertenecientes a otros clústeres temáticos, incluyendo algunos que tienen relaciones directas con los nodos del primero. No obstante, resulta fundamental analizar estas otras relaciones puesto que hacen visibles diferentes temáticas relevantes para el subcampo y estrechamente vinculadas con él, en procesos de diálogo disciplinar.

\footnotetext{
${ }^{5}$ Igualmente debe hacerse notar el elevado número de documentos (23) en los que no se especifica el país de los autores.
}

Esta red "extendida" es precisamente la que aquí se ha denominado como el Entramado Temático de la Comunicación Organizacional.

$\mathrm{Y}$ es que, desde la óptica estructural, la densidad o cohesión de una red dada por sus relaciones hacia dentro, de carácter homófilo (su bonding) es positiva, ya que permite establecer límites conceptuales y es fuente de coherencia y estabilidad interna; pero al mismo tiempo, su apertura o vinculación con otras redes dada por relaciones más heterófilas (su bridging) es también positiva, ya que son estos "puentes" los que posibilitan, por ejemplo, el acceso a información diferente y no redundante, la diversidad y el establecimiento de mecanismos dialógicos y colaborativos $^{6}$ (García-Macías, 2015; Lozares, Martí, Molina, \& García-Macías, 2013; Lozares \& Verd, 2011).

En primer lugar, están las relaciones con el resto de las palabras clave ( 24 adicionales, en negritas en la Tabla 4) de los 128 artículos de los que emerge el Clúster CCO, con las cuales evidentemente hay co-ocurrencia. Después están

\footnotetext{
${ }^{6}$ Estas discusiones sobre cohesión - vinculación, homofilia - heterofilia o bonding - bridging han sido ampliamente abordadas en la literatura clásica sobre redes sociales, por ejemplo, en las formulaciones de la fortaleza de los lazos débiles de Granovetter (1973); los agujeros estructurales de Burt (1992, 2004) o las proposiciones de Capital Social de Lin (2001, 2008).
} 
Tabla 4. Palabras directamente relacionadas con los nodos del Clúster Conceptual de Comunicación Organizacional, según su clúster de origen en la red completa.

\begin{tabular}{|c|c|c|c|c|}
\hline \multicolumn{5}{|c|}{ Clúster } \\
\hline 1 & 2 & 3 & 4 & 6 \\
\hline Activismo & Aprendizaje & Acontecimiento & Ciencias sociales & Arte \\
\hline Chile & Cambio social & Audiencias & Concentración mediática & Diseño \\
\hline Comunicación financiera & Ciencia & Comunidades virtuales & Crisis & Ética de los negocios \\
\hline Comunicación política & Comunicación & Convergencia & Ecología de los medios & Identidad \\
\hline Comunicación pública & Com. para el cambio social & Cultura & Fenomenología & Imagen \\
\hline Contenidos & Com. para el desarrollo & Cultura organizacional & Persuasión & Lenguaje \\
\hline Control & Comunicación participativa & Discurso & Políticas públicas & Moda \\
\hline Democracia & Conflicto & Estrategia & Proceso de comunicación & Publicidad \\
\hline Democratización & Consumo & Fragmentación & Semiótica & \\
\hline Engagement & Desarrollo & Globalización & Sustentabilidad & \\
\hline España & Desarrollo sostenible & Historia & Teoría crítica & \\
\hline Ética profesional & Educación superior & Inteligencia colectiva & Teoría de la comunicación & \\
\hline Etnografía & Educomunicación & Interacciones & & \\
\hline Facebook & Enseñanza & Interactividad & & \\
\hline Framing & Estado del arte & Planificación & & \\
\hline Internet & Estrategias de comunicación & Poder & & \\
\hline Marketing político & Formación profesional & Subjetividad & & \\
\hline Mediaciones & Gestión del conocimiento & Tecnologías & & \\
\hline Medios de comunicación & Investigación & Web 2.0 & & \\
\hline Movimientos sociales & Jóvenes & & & \\
\hline Opinión pública & Medio ambiente & & & \\
\hline Periodistas & Paradigmas & & & \\
\hline Prensa & Participación & & & \\
\hline Redes sociales & Sociedad de la información & & & \\
\hline Sociedad & Tecnologías de la inf. y la com. & & & \\
\hline Transparencia & Universidad & & & \\
\hline Twitter & & & & \\
\hline Youtube & & & & \\
\hline
\end{tabular}

Fuente: Elaboración propia. Palabras con una ocurrencia mínima de 3 (n=93). Se excluye el clúster 5 que corresponde a Comunicación Organizacional. Las 24 palabras en negritas pertenecen a los 128 documentos que dan origen al componente aislado. El resto (69) pertenecen a otros documentos de la red completa.

otras 69 palabras directamente relacionadas con las del clúster, que provienen del resto de los artículos del corpus. Estos 93 nuevos nodos, que se presentan en la Tabla 4, se agregan a los 37 originales para conformar una nueva red compuesta por 130 temáticas.

Los términos Comunicación y Medios de Comunicación destacan por su elevada frecuencia de apariciones, de 176 y 99 ocasiones respectivamente. Aunque el resto se ubican muy por debajo de ese rango, con menos de 50 ocurrencias, se observa una serie de temas con claro protagonismo, como Tecnologías de la información y la comunicación, Redes Sociales, Internet, Cultura, Comunicación política, Comunicación para el desarrollo y Tecnologías.

La Figura 3 es el sociograma de este entramado temático ampliado, en el que se representa la importancia relativa de los conceptos de acuerdo con el atributo Frt en la red completa. En él se aprecian claramente los temas más próximos al Clúster $\mathrm{CCO}$, destacando por supuesto los de índole mediático en general y especialmente los medios sociales (Facebook, Youtube, Twitter), pero además otras preocupaciones de carácter eminentemente social (Comunicación para el desarrollo, Cambio social, Desarrollo sostenible, Movimientos sociales, Democracia, Poder), teórico-metodológico (Investigación, Enseñanza, Ciencias sociales, Fenomenología) y aplicativo (Publicidad, Diseño, Moda). Vale destacar la preminencia del concepto Redes sociales que, como se describirá más adelante, en efecto adquiere una importancia central.

Una variante de visualización del entramado se presenta en la Figura 4, a manera de un grafo de densidad que denota diversas áreas importantes de concentración y en el que se perciben algunos conceptos diferentes a los anteriormente descritos: se aprecia la densidad en torno a temas como Relaciones Públicas, Comunicación empresarial, Comunicación corporativa, Reputación corporativa, Gestión de la Comunicación, Redes sociales, Comunicación y 


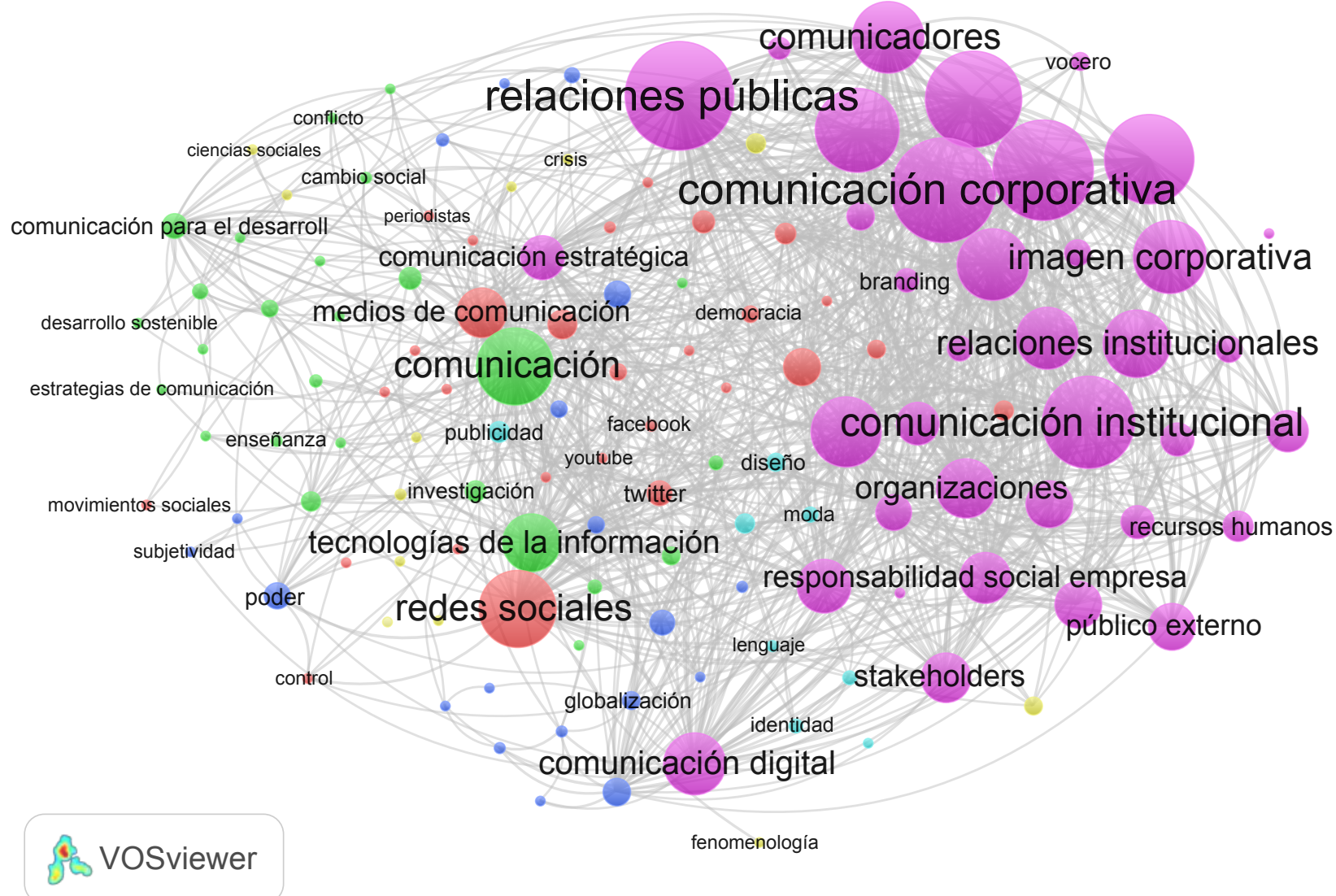

Figura 3. Importancia relativa de los nodos del Entramado Temático de la Comunicación Organizacional, según la fortaleza de sus lazos.

Tecnologías. Estas áreas con mayor intensidad de color pueden dar pistas visuales sobre los temas centrales o core issues del subcampo.

\section{Los temas centrales del Entramado}

Previamente se argumentó que, a través del ARS y sus métricas estructurales, es posible analizar la importancia de los nodos individuales en las redes, más allá de los tradicionales recuentos de frecuencias nominales de los temas. Hasta ahora, junto a esos recuentos, se han introducido algunos indicadores relacionales como atributos de los nodos (e.g., Ocr, Vnc y Frt en la Tabla 1), por lo que a continuación se recurre al concepto de centralidad, que es uno de los fundamentales en el ARS. La centralidad de un nodo es un indicador de la importancia que este adquiere por sus vínculos y su posición en la red. Para efectos de este análisis se calculan tres tipos de centralidad, considerados prototípicos (Schoch \& Brandes, 2016): el grado (degree), la cercanía (closeness) y la intermediación (betweeness).

El grado indica qué tan bien conectado está un nodo en su entorno y se expresa como el número de nodos con los cuales es adyacente o está directamente ligado, por lo que un mayor grado significa que un actor es más central en la red; la cercanía indica que un nodo es más central en tanto se encuentre a cortas distancias de los otros, que sea más fácilmente "accesible" y por tanto se expresa como la suma de las distancias geodésicas con el resto de los nodos en el grafo; y finalmente, la intermediación mide hasta qué punto un nodo se encuentra más "entre" los otros, ubicado en los caminos más cortos, es decir, es el número de veces que un actor necesita de otro, para alcanzar a cualquiera otro (Borgatti \& Everett, 2006; Freeman, 1979; Scott, 2000). 


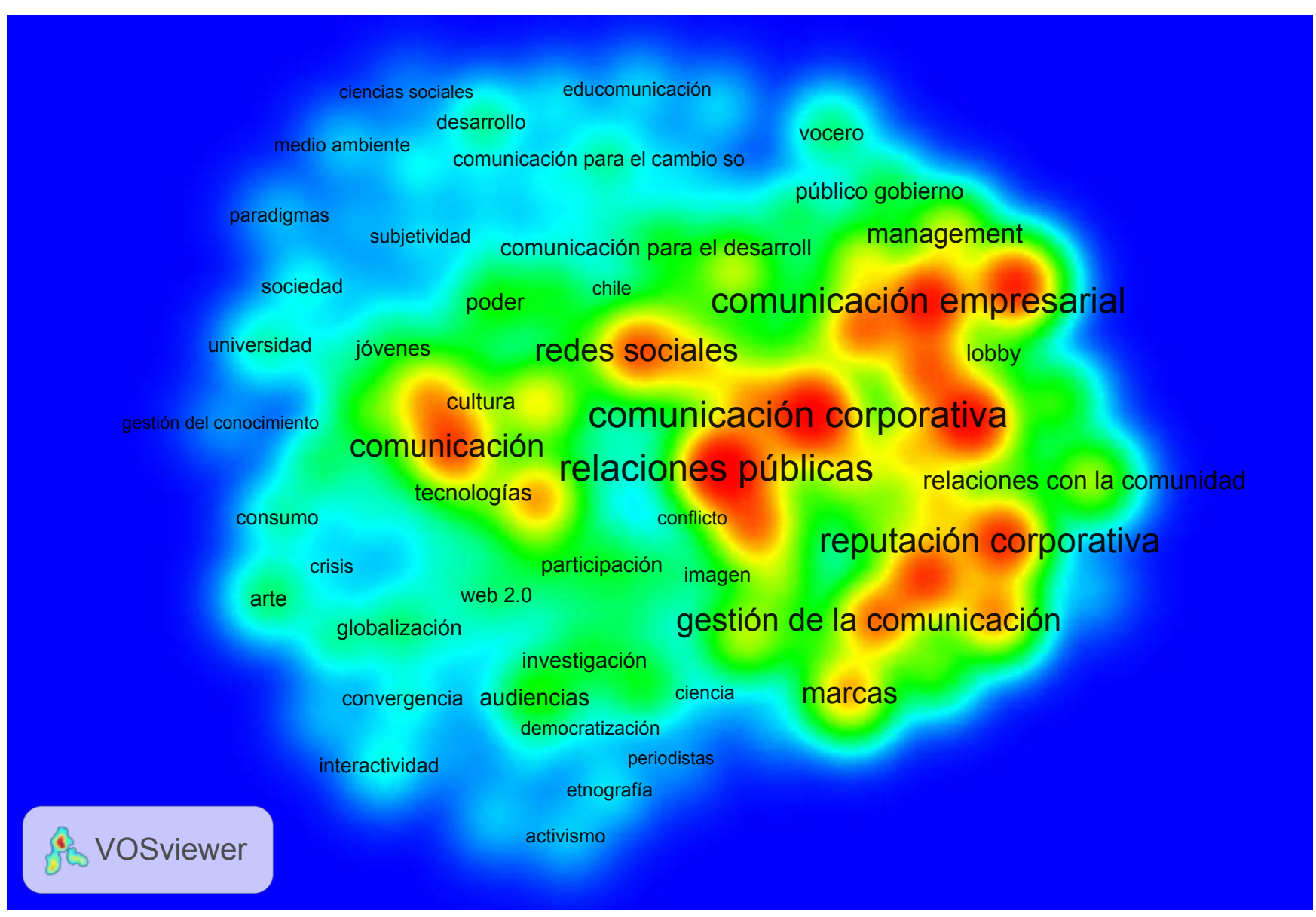

Figura 4. Grafo de densidad del Entramado Temático de la Comunicación Organizacional. El tamaño de las etiquetas indica la fortaleza de lazo de cada concepto y la intensidad del color representa la concentración conceptual según co-ocurrencia de palabras clave.

Resulta evidente que, si bien remiten a un concepto común, las tres medidas difieren entre sí. Por ejemplo, un actor puede tener muy pocas conexiones (grado bajo), pero estar ubicado de manera que la distancia promedio a los demás nodos sea muy corta y por lo tanto se requieran de pocos intermediarios para alcanzarlo (alta centralidad) o bien, puede fungir como elemento conector entre dos segmentos de la red que, en su ausencia, estarían desconectados (alta intermediación).

En la Tabla 5 se presentan, en la forma de rankings de veinte posiciones, las palabras clave con los más altos niveles en cada tipo de centralidad en el Entramado Temático. Considerando que algunos niveles son ocupados por más de un concepto cuando se presentan puntuaciones idénticas, en total aparecen 39 diferentes palabras clave, aunque solo 14 se mantienen constantes en los tres listados. La columna de la centralidad de grado contiene 33 términos distintos, la de cercanía 30 y la de intermediación 20, ya que en este caso se trata de puntuaciones únicas.

Del total de 39 temas diferentes, 27 pertenecen de origen al Clúster CCO, mientras que 12 (marcados en la tabla con tipografía en negritas) son parte de los conceptos relacionados que fueron incluidos en la segunda ola. Cuatro de estos últimos aparecen con una centralidad muy alta, ya que se ubican entre los primeros lugares de los tres rankings: Comunicación, Redes sociales, Medios de comunicación y Tecnologías de la información y la comunicación.

Con posiciones menos relevantes, tanto en grado como en cercanía, aparecen Comunicación política y Audiencias, mientras que en el ranking de intermediación emergen seis palabras que no están incluidas en los otros dos: Participación, 
Tabla 5. Rankings a 20 posiciones de los indicadores de centralidad de las palabras clave del Entramado Temático de la Comunicación Organizacional.

\begin{tabular}{|c|c|c|c|c|c|c|c|c|}
\hline \multicolumn{3}{|c|}{ Centralidad de Grado } & \multicolumn{3}{|c|}{ Centralidad de Cercanía } & \multicolumn{3}{|c|}{ Centralidad de Intermediación } \\
\hline RK & Palabra Clave & $(\%)$ & RK & Palabra Clave & $(\%)$ & RK & Palabra Clave & $(\%)$ \\
\hline 1 & Comunicación & 2.5393 & 1 & Comunicación & 1.0177 & 1 & Comunicación & 14.3256 \\
\hline \multirow[t]{2}{*}{2} & Redes sociales & \multirow{2}{*}{2.4678} & \multirow{2}{*}{2} & Relaciones públicas & \multirow{2}{*}{1.0123} & 2 & Medios de comunicación & 8.1384 \\
\hline & Relaciones públicas & & & Redes sociales & & 3 & Relaciones públicas & 6.8663 \\
\hline 3 & Medios de comunicación & 2.2175 & \multirow{3}{*}{3} & Comunicación corporativa & \multirow{3}{*}{0.9663} & 4 & Redes sociales & 6.3613 \\
\hline \multirow{2}{*}{4} & Tecnologías de la inf. y la com. & \multirow{2}{*}{2.1459} & & Tecnologías de la inf. y la com. & & 5 & Tecnologías de la inf. y la com. & 5.7809 \\
\hline & Comunicación corporativa & & & Medios de comunicación & & 6 & Comunicación digital & 4.2503 \\
\hline \multirow{2}{*}{5} & Comunicación organizacional & \multirow{2}{*}{1.9671} & \multirow{2}{*}{4} & Organizaciones & \multirow{2}{*}{0.9425} & 7 & Comunicación organizacional & 3.7324 \\
\hline & Organizaciones & & & Comunicación organizacional & & 8 & Organizaciones & 3.1870 \\
\hline 6 & Medios sociales & 1.8956 & 5 & Medios sociales & 0.9333 & 9 & Medios sociales & 3.1812 \\
\hline \multirow{2}{*}{7} & Comunicación empresarial & \multirow{2}{*}{1.8598} & \multirow{2}{*}{6} & Comunicación empresarial & \multirow{2}{*}{0.9288} & 10 & Comunicación estratégica & 3.0254 \\
\hline & Públicos & & & Públicos & & 11 & Comunicación corporativa & 3.0117 \\
\hline 8 & Dircom & 1.8240 & 7 & Dircom & 0.9243 & 12 & Resp. social empresarial & 1.5957 \\
\hline 9 & Reputación corporativa & 1.7525 & 8 & Reputación corporativa & 0.9155 & 13 & Participación & 1.5017 \\
\hline 10 & Gestión de la comunicación & 1.7167 & 9 & Gestión de la comunicación & 0.9111 & 14 & Internet & 1.4958 \\
\hline \multirow{3}{*}{11} & Comunicación institucional & \multirow{3}{*}{1.6810} & \multirow{2}{*}{10} & Comunicación institucional & \multirow{2}{*}{0.9068} & 15 & Comunicación para el desarrollo & 1.3864 \\
\hline & Comunicación digital & & & Comunicación estratégica & & 16 & Públicos & 1.3695 \\
\hline & Comunicación estratégica & & \multirow{2}{*}{11} & Imagen corporativa & \multirow{2}{*}{0.9025} & 17 & Marcas & 1.3093 \\
\hline 12 & Imagen corporativa & 1.6452 & & Comunicación digital & & 18 & Poder & 1.2474 \\
\hline \multirow{4}{*}{13} & Comunicadores & \multirow{4}{*}{1.6094} & & Marcas & & 19 & Prensa & 1.2335 \\
\hline & Marcas & & 12 & Resp. social empresarial & 0.8983 & 20 & Tecnologías & 1.1647 \\
\hline & Comunicación interna & & 13 & Comunicadores & 08041 & & & \\
\hline & Resp. social empresarial & & 13 & Comunicación interna & 0.8941 & & & \\
\hline 14 & Stakeholders & 1.5021 & 13 & Stakeholders & 0.8858 & & & \\
\hline 15 & Marketing & 1.4664 & 14 & Marketing & 0.8817 & & & \\
\hline 16 & Comunicación política & 1.4306 & 15 & Comunicación política & 0.8697 & & & \\
\hline 17 & Relaciones institucionales & 1.3591 & 16 & Relaciones institucionales & 0.8658 & & & \\
\hline 18 & Management & 1.3233 & 17 & Management & 0.8580 & & & \\
\hline & Público externo & & & Relaciones con la prensa & & & & \\
\hline & Público interno & & 18 & Relaciones con los medios & 0.8542 & & & \\
\hline 19 & Relaciones con la prensa & 1.2518 & 19 & Audiencias & 0.8504 & & & \\
\hline & Relaciones con los medios & & 20 & Público externo & 0.8466 & & & \\
\hline 20 & Audiencias & 12160 & & & & & & \\
\hline 20 & Gobierno corporativo & 1.2160 & & & & & & \\
\hline
\end{tabular}

Fuente: Elaboración propia. Cálculos con base en la red de 130 nodos conformada por el clúster de Comunicación Organizacional y las palabras de otros clústeres directamente relacionadas. Los puntaje similares se tratan como la misma posición en el ranking. Se identifican con negritas las palabras que originalmente no pertenecían al Clúster Conceptual.

Internet, Comunicación para el desarrollo, Poder, Prensa y Tecnologías.

El hecho de que estos conceptos de la segunda ola posean niveles de centralidad tan destacados significa que ocupan espacios muy cercanos de diálogo disciplinar con los temas del Clúster CCO, contribuyendo a posicionar los core issues del Entramado Temático de la Comunicación Organizacional e inclusive constituyendo otros más.

Con respecto a los temas originales del Clúster CCO, el término Relaciones públicas se ubica en posición predominante (es segundo en grado y cercanía, y tercero en intermediación); Comunicación corporativa es cuarto en los dos primeros rankings, aunque desciende hasta el onceavo en intermediación; Comunicación organizacional, Organizaciones, Comunicación digital, Medios sociales, Comunicación empresarial, Públicos,
Dircom y Comunicación estratégica también tienen posiciones muy destacadas.

En cambio, diez de los conceptos cuya frecuencia de ocurrencias era muy baja en el Clúster $\mathrm{CCO}$, no alcanzan a insertarse entre los veinte más centrales en ninguna de las categorías, hecho que confirma que desempeñan un papel secundario en el Entramado. Se trata de Evaluación, Relaciones con la comunidad, Branding, Lobby, Liderazgo, Recursos humanos, Tendencias, Público gobierno, Vocero e Identidad corporativa.

\section{Conclusiones}

A partir de los datos relacionales de la producción científica en comunicación contenida en el SciELO Citation Index, en este artículo se identificó y describió, desde una perspectiva estructural y a través de los métodos del 
Análisis de Redes Sociales, el Entramado Temático de la Comunicación Organizacional. Se trata de una red compuesta por 130 conceptos vinculados entre sí, de los cuales (a) 37 pertenecen al denominado Clúster Conceptual de la Comunicación Organizacional y; (b) 93 provienen de otros clústeres de la más amplia red conceptual del campo de la comunicación, pero están directamente vinculados con los nodos del primero.

Esta doble constitución del entramado posibilitó una forma de análisis dual que atiende, en principio, al núcleo temático duro de la comunicación organizacional junto con la producción académica específica que lo sustenta y, posteriormente, a sus interacciones directas con temas y objetos de estudio propios de otras subespecialidades de la comunicación. De todo ello se derivan algunos elementos conclusivos:

1. Dentro de la red conceptual del campo de la comunicación en general, que por su configuración puede catalogarse como heterogénea y dispersa, el conglomerado de la comunicación organizacional es el componente más nítidamente diferenciado.

2. Este componente, el Clúster $\mathrm{CCO}$, que proviene específicamente de un subconjunto de 128 documentos, muestra tres claros factores de concentración de la producción académica: (1) uno de tipo editorial, ya que casi tres cuartas partes de los artículos fueron publicados por únicamente cuatro revistas; (2) otro de carácter nacional, en virtud de que en tan solo cuatro países se produjo alrededor del 70 por ciento de los artículos referidos; y (3) uno más de naturaleza institucional, dado que uno de cada cinco textos fue generado por investigadores pertenecientes a tan solo tres universidades (que también auspician las revistas con mayor número de publicaciones).

3. En el Clúster CCO, además de que se identifican con facilidad los temas centrales o core issues, también hay elementos concluyentes para establecer que se trata de una red muy cohesiva internamente; esto es, que los temas que la conforman están fuertemente vinculados entre sí, debido a sus co-ocurrencias. Se trata de un bloque con solidez y coherencia temática, que son importantes propiedades bonding.
4. Paralelamente, varios de los nodos relevantes del Clúster $\mathrm{CCO}$ se relacionan directa, e incluso fuertemente, con otros conceptos de diferentes subdisciplinas de la comunicación. Al considerar todo el conjunto como una nueva red, se observa que los conceptos recién añadidos se posicionan de forma estratégica y adquieren niveles de centralidad muy elevados. De ello se deduce que, aunque el Clúster $\mathrm{CCO}$ es un bloque sólido y cohesivo, de ninguna manera se encuentra aislado, sino que tiene múltiples e importantes canales de diálogo con otras temáticas del campo, gracias a que algunos de sus core issues son puentes naturales de colaboración disciplinar. Indudablemente, varias de estas temáticas "externas" son transversales en el campo, con lo que, en realidad, llegan a ser muy centrales para la comunicación organizacional, al igual que lo son para otras áreas. El subcampo, entonces, tiene también importantes propiedades bridging.

Con todo y su valor ilustrativo, estas conclusiones deben ser contextualizadas conforme a los alcances y limitaciones del estudio: son válidas con respecto al conjunto de datos bibliográficos utilizado, que se reconoce que puede presentar algunas limitaciones de origen; por ejemplo, el hecho de que algunos artículos relevantes efectivamente contenidos en SciELO no formen parte de la base de datos del estudio, debido a que no fueron indexados bajo el tema Comunicación, el primer criterio selectivo utilizado; o bien, que la limpieza y corrección manual de las inconsistencias de la base de registros no hayan sido suficientes. Estas inconsistencias son muy frecuentes debido a la obtención y el tratamiento automatizado de los registros, entre otros problemas relacionados con los bancos de datos en línea (Montoya et al., 2018).

En cualquier caso, se considera que este ejercicio analítico y descriptivo constituye un primer acercamiento al subcampo de la comunicación organizacional a través de su producción científica y desde una perspectiva netamente relacional, lo que puede contribuir a detonar procesos reflexivos posteriores más extensos, no solo sobre sus contenidos temáticos, sino también sobre otras múltiples configuraciones estructurales que intrigan e interesan a los académicos y practicantes de la comunicación, especialmente de la organizacional. 


\section{Referencias}

Borgatti, S. P., \& Everett, M. G. (2006). A Graph-theoretic perspective on centrality. Social Networks, 28(4), 466-484.

Brandes, U., Kenis, P., \& Raab, J. (2005). La explicación a través de la visualización de redes. REDES. Revista Hispana Para El Análisis de Redes Sociales, 9(6), 1-19.

Burt, R. S. (1992). Structural holes: the social structure of competition. Cambridge: Harvard University Press.

Degene, A., \& Forsé, M. (1999). Introducing Social Networks. London, Thousand Oaks, New Delhi: SAGE Publications Inc.

Freeman, L. C. (1979). Centrality in social networks 1: conceptual clarification. Social Networks, 1(3), 215-239.

Fuentes-Navarro, R. (2016). Implicaciones de una «nueva» historia (internacional) de la institucionalización de los estudios de la comunicación en América Latina. En E. Vizer \& C. Vidales (Eds.), Comunicación, campo(s), teorías y problemas. Una perspectiva internacional. Salamanca: Comunicación Social ediciones y publicaciones.

García-Macías, A. (2015). Capital social relacional en enclaves de la industria del vestido en México. Tesis Doctoral. Universidad Autónoma de Barcelona.

García-Macías, A., \& Fortanell, B. (2018). El "mundo pequeño" de la investigación sobre comunicación en América Latina. Aproximaciones desde el Análisis de Redes Sociales a las publicaciones científicas de la disciplina en el SciELO Citation Index. XXIX Encuentro Nacional AMIC 2018, Monterrey, Nuevo León, México.

Granovetter, M. (1973). The strength of weak ties. American Journal of Sociology, 78(6), 1360-1380.

Gutiérrez-Salcedo, M., Martínez, M. Á., Moral-Munoz, J. A., Herrera-Viedma, E., \& Cobo, M. J. (2017). Some bibliometric procedures for analyzing and evaluating research fields. Applied Intelligence, 1-13.

Khasseh, A. A., Soheili, F., Moghaddam, H. S., \& Chelak, A. M. (2017). Intellectual structure of knowledge in iMetrics: A co-word analysis. Information Processing and Management, 53(3), 705-720.

Knoke, D., \& Yang, S. (2008). Social Network Analysis (2nd ed.). Los Angeles, London, New Delhi, Singapore: SAGE Publications Inc.

Lin, N. (2001). Social Capital: A Theory of Social Structure and Action. Cambridge University Press.

Lin, N. (2008). A Network Theory of Social Capital. In D. Castiglione, J. Van Deth, \& G. Wolleb (Eds.), The Handbook of Social Capital (pp. 50-59). Oxford University Press.

Lozares, C. (1996). La teoría de Redes Sociales. Papers, 48, 103-126.
Lozares, C. (2005). Bases socio-metodologicas para el Análisis de Redes Sociales ARS. EMPIRIA. Revista de Metodología de Ciencias Sociales, (10, Julio-diciembre 2005), 9-35.

Lozares, C., Martí, J., Molina, J. L., \& García-Macías, A. (2013). La cohesión-integración versus la fragmentación social desde una perspectiva relacional. Metodología de Encuestas, 15, 57-75.

Lozares, C., \& Verd, J. M. (2011). De la Homofilia a la Cohesión social y viceversa. REDES-Revista Hispana Para El Análisis de Redes Sociales, 20(2).

Montoya, F. G., Alcayde, A., Baños, R., \& Manzano-Agugliaro, F. (2018). A fast method for identifying worldwide scientific collaborations using the Scopus database. Telematics and Informatics, 35(1), $168-185$.

Schoch, D., \& Brandes, U. (2016). Re-conceptualizing centrality in social networks. European Journal of Applied Mathematics, 27(6), 971-985.

SciELO. (n.d.). Sobre el SciELO: Modelo SciELO. Retrieved April 30, 2018, from http://www.scielo.org

Scott, J. (2000). Social Network Analysis. A Handbook. (2nd ed.). London, Thousand Oaks, New Delhi: SAGE Publications Inc.

van Eck, N. J., \& Waltman, L. (2013). VOSviewer Manual. 1 January 2013, (January), 1-28.

van Eck, N. J., \& Waltman, L. (2014). Visualizing Bibliometric Networks. Measuring Scholarly Impact. http://doi.org/ 10.1007/978-3-319-10377-8_13

van Eck, N. J., Waltman, L., Dekker, R., \& van den Berg, J. (2010). A Comparison of TwoTechniques for Bibliometric Mapping: Multidimensional Scaling and VOS. Journal of the American Society for Information Science and Technology, 61(12), 2405-2416.

Wasserman, S., \& Faust, K. (1994). Introduction to Social Network Analysis. Cambridge; New York: Cambridge University Press.

Zhao, W., Mao, J., \& Lu, K. (2018). Ranking themes on co-word networks: Exploring the relationships among different metrics. Information Processing and Management, 54(2), 203-218. 